\title{
A CASE OF STAPHYLOCOCCAL ENDOCARDITIS
}

J. Horton, M.B., Сн.B.

Late Senior House Officer, University Department of Medicine, The Royal Hospital, Sheffield

\section{Introduction}

Staphylococcal endocarditis has a very high mortality rate ${ }^{3,5}$ and difficulties in its therapy have not been lessened by the emergence of strains of the organism resistant to the commonly used antibiotics. Recently, encouraging reports of the use of vancomycin in resistant staphylococcal infections have appeared.1, 2, 4 Since the number of patients treated is small, the following case report is thought to be of interest.

\section{Case Report}

A 16-year-old G.P.O. messenger boy was involved in a motor-cycle accident on May 24, 1959, and was admitted to a surgical unit at the Royal Hospital, Sheffield. Examination revealed a small but well-developed boy who was passing bright red blood per urethram. The pulse was regular, the rate 96 per minute, the blood pressure 100/65 $\mathrm{mm}$. $\mathrm{Hg}$. and temperature $98^{\circ} \mathrm{F}$. There was bruising of the left side of the forehead, a $\mathrm{r}$-in. long laceration just above the left eye and tenderness over the lower lumbar spine. Examination of the chest was entirely normal and no heart murmurs were heard. The abdomen was rather tense, but was not tender. Before the accident the patient had always enjoyed good health and had never suffered from rheumatic fever or chorea. An X-ray of the lumbar spine showed an impacted fracture of the fourth lumbar vertebra. An X-ray of the skull was normal. After an unsuccessful attempt to pass a catheter into the bladder, a diagnosis of ruptured urethra was made. At operation the rupture, which was found to involve the membranous urethra, was repaired around a catheter inserted through the bladder via a suprapubic incision. During the operation the patient was transfused two pints of blood. Post-operatively he was given penicillin, 500,000 units sixhourly by intramuscular injection, and sulphadimidine, I g. six-hourly by mouth. The catheter was removed on the tenth day after admission and the patient became febrile for the first time (see figure). Examination of the chest was unremark- able, but pus cells were found in the urine and $\vec{\omega}$ culture yielded a Staphylococcus pyogenes which could not be typed with any phage. The organism was highly resistant to penicillin, sulphadimidine and tetracycline, but was sensitive to chloro- ? mycetin, erythromycin and novobiocin. On the $\vec{\overrightarrow{ }}$ tenth day after admission the sulphadimidine was $\infty$ discontinued and replaced by streptomycin, $0.5 \mathrm{~g}$. b.d. by intramuscular injection. The patient had 0 continued to have haematuria since admission and because of a fall in haemoglobin concentration from $13.9 \mathrm{~g}$. per $100 \mathrm{ml}$. on admission to $9.9 \mathrm{~g}$. per $100 \mathrm{ml}$. the patient was transfused with one $\frac{c}{\omega}$ pint of whole blood, during which he suffere $\overrightarrow{\vec{\theta}}$ several rigors. The haematuria persisted and the patient developed a clot retention on the $17 \mathrm{th}$. day after admission, which was treated success fully by irrigation of the bladder. Because of $\underset{a}{a}$ further fall in the haemoglobin concentration the patient was transfused with four more pints of $\stackrel{0}{2}$ whole blood. Despite the antibiotic therapy, the $\stackrel{\mathbb{Q}}{2}$ fever persisted and on the 2 ist day after admission $\overrightarrow{\overrightarrow{0}}$ increased markedly and the patient had several $\exists$ rigors. Following this, he complained of pain in his left wrist and shoulder and both joints were found to be swollen, warm and tender with limitation of movements. The spleen was now palpable on abdominal examination. A blood culture was taken and this yielded a heavy pure growth of 3 . Staphylococcus pyogenes which could not be typed of with any phage. This organism was sensitive to the same antibiotics as the one previously isolated 의 from the urine, which was now sterile. On the $D$ 22nd day after admission the penicillin and streptomycin were discontinued and chloramphenicol was $N$ substituted in a dosage of $\mathrm{I} \mathrm{g}$. six-hourly for the first 24 hours and 0.75 g. six-hourly thereafter $\tilde{N}$ (Fig. I).

Although the pain and swelling of his joints subsided, the spleen remained palpable and $24 \circ$ days after admission an aortic diastolic cardiac $\overparen{\Phi}$ murmur was heard for the first time. This murmur $\stackrel{\oplus}{?}$ gradually became louder, but no haemorrhages $T$ were found in the skin or mucous membranes 


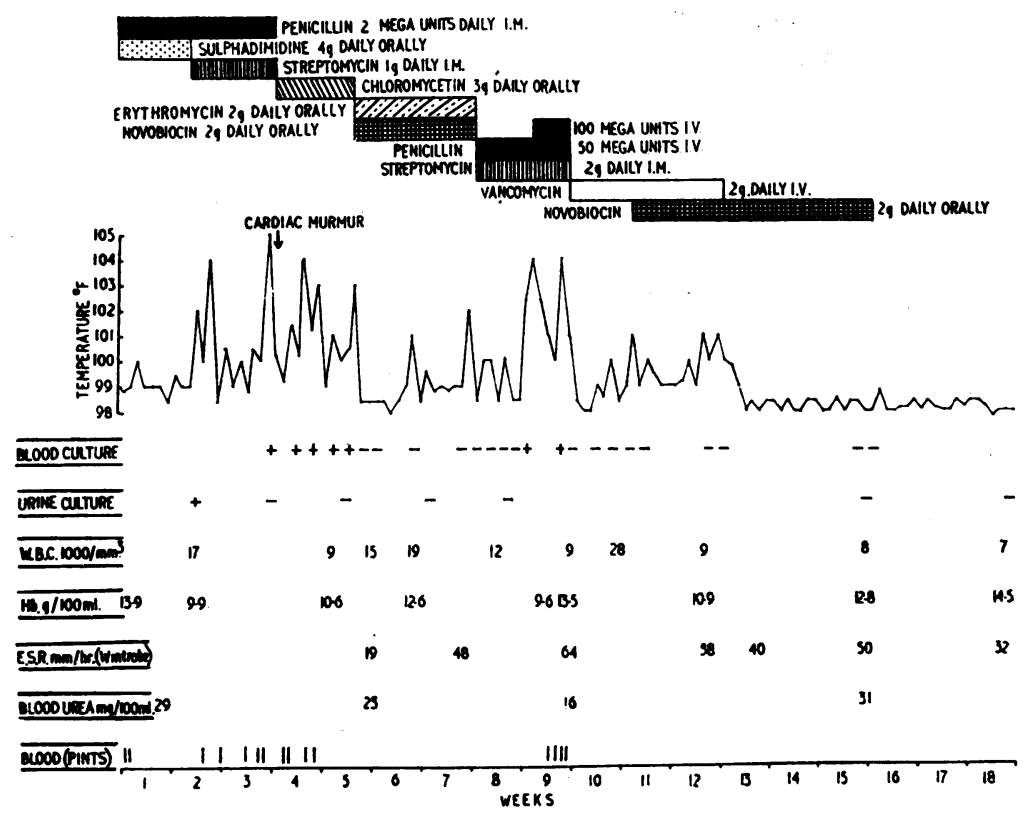

FIG. I

and the urine contained neither red blood cells nor casts. The chloramphenicol was replaced first by a combination of erythromycin, 0.5 g. six-hourly, and novobiocin, $0.5 \mathrm{~g}$. six-hourly, by mouth and then by a combination of streptomycin, $1 \mathrm{~g}$. twice daily by intramuscular injection and penicillin in a dosage of first 50 mega units daily and then 100 mega units daily (Fig. I). The latter was given as a continuous infusion through a polythene catheter introduced into an antecubital vein and passed as far as the left innominate vein. The endocarditis, however, remained active and continuing anaemia necessitated further blood transfusion.

Sixty-one days after admission a supply of vancomycin was obtained and the penicillin and streptomycin discontinued (Fig. I). The vancomycin was given in a dose of $2 \mathrm{~g}$. daily by continuous intravenous infusion through the polythene catheter. The organism was reported to be sensitive to vancomycin at a level of $2.5 \mu \mathrm{g}$. per $\mathrm{ml}$. and the serum vancomycin level was maintained at approximately ro $\mu \mathrm{g}$. per $\mathrm{ml}$. Initially the temperature returned towards normal, but after four days' therapy it again began to rise and a low-pitched mid-diastolic murmur was heard just within the cardiac apex. Blood cultures at this time and subsequently, however, were sterile and all traces of blood disappeared from the urine. The vancomycin gave rise to a marked phlebitis and the position of the catheter had to be changed on two occasions. Although it was thought that this recurrent phlebitis was mainly responsible for the continued fever, he was again given novobiocin, $0.5 \mathrm{~g}$. six-hourly by mouth, in addition to the vancomycin. The leucocyte count had risen to 28,000 per cu. mm., of which $65 \%$ were neutrophils, but there was no clinical or radiological evidence of abscess formation, either in the pelvis or in the perinephric region. The vancomycin was discontinued after having been given continuously for 23 days. The patient was now allowed up and, as the inflammatory reaction around the site of the tip of the polythene catheter subsided, the temperature fell to normal. Examination of the heart showed that the apical mid-diastolic murmur was no longer audible, though the aortic diastolic murmur persisted unchanged. The spleen was still palpable. The haemoglobin was i I g. per $100 \mathrm{ml}$., the leucocyte count 9,000 per cu. mm. with $65 \%$ neutrophil polymorphs, and the E.S.R. 40 $\mathrm{mm}$. in one hour (Wintrobe). The novobiocin was continued for a further three weeks, after which the patient continued to be afebrile. When he was discharged from hospital, four months after admission, the patient had no symptoms and his weight had increased by $14 \mathrm{lb}$. over the lowest weight recorded in hospital. Examination of the heart showed that the aortic diastolic murmur was still present, but there was no evidence of cardiac failure. The spleen was still palpable, but was no longer tender. The urethra and fractured lumbar vertebra were well healed. Chest X-ray and electrocardiograms were normal. The haemoglobin was 14.5 g. per $100 \mathrm{ml}$., leucocyte count 7,000 
per cu.mm., E.S.R. $32 \mathrm{~mm}$. in one hour (Wintrobe) and the urine was entirely normal.

\section{Discussion}

The endocarditis in the present patient followed infection of the urinary tract. This sequence would appear to be not uncommon, as it occurred in eight of the 38 cases of staphylococcal endocarditis reported by Fisher et al. ${ }^{3}$ Although apparently adequate blood levels of chloromycetin were obtained after the initial diagnosis of septicaemia was made, the patient's temperature was unaffected and blood cultures were repeatedly positive. It was thus apparent that this antibiotic had little or no effect on the infection. Similarly, although erythromycin and novobiocin controlled the infection as judged by negative blood culture, the patient's condition deteriorated and that the infection remained active was shown by the recurrence of positive blood cultures after these antibiotics were stopped. Fisher et al. ${ }^{3}$ considered that penicillin in large doses, despite in vitro resistance of the organism, was the most effective form of treatment and Melton and Logue ${ }^{6}$ reported cure of eight out of nine patients using a combination of penicillin and streptomycin supplemented occasionally by one of the newer anti-microbial agents. Despite very large doses of penicillin, in excess of those commonly used by these groups of workers, in combination with streptomycin, the patient's condition only improved for a short time before a further relapse took place and blood cultures again became positive. After these antibiotics had failed to control the infection vancomycin was commenced. The patient's condition improved dramatically and, despite a recurrence of fever, due probably to phlebitis round the tip of the catheter, all subsequent blood cultures remained sterile. It thus seems reasonable to ascribe the successful eradication of the infection to this antibiotic.

The successful treatment of the staphylococcal endocarditis in the present patient by vancomycin is in keeping with the experience of Geraci et al.,4 who reported the cure of five out of six patients with staphylococcal endocarditis after treatment with this antibiotic. The only toxic effect of the drug noted in this patient was phlebitis, which developed around the tip of the polythene catheter and despite the large total dose of $46 \mathrm{~g}$. of vancomycin, there was no evidence of deafness which previous workers have noted in several patients. ${ }^{2,4}$ These, however, all had impaired renal function; despite a ruptured urethra and clot retention, renal impairment was never apparent in this patient.

It is suggested that vancomycin is probably the drug of choice in endocarditis due to penicillinresistant staphylococci. Except for the production of phlebitis, it appears to be relatively nontoxic in patients with normal renal function.

\section{Acknowledgments}

I thank Professor C. H. Stuart Harris and Dr. J. D. S. Hammond for their help and encouragement, and Professor C. H. Stuart Harris for permission to report this case.

\section{REFERENCES}

I. DAVIS, A., RAMSEY, A. M., and MANN, A. J. (1958) Brit. med. F., il, 1394.

2. DUTTON, A. A. C., and ELMES, P. C. (1959), Ibid., i, Ir44

3. FISHER, A. M., WAGNER, H. N., and ROSS, R. D. (1955), Arch. intern. Med., 95, 427-437.

4. GERACI, J. E., HEILMAN, F. R., NICHOLS, D. R., and WELLMAN, W. E. (1958), Proc. Mayo Clin., 33, 172.

5. GERACI, J. E. (1958), Med. Clin. N. Amer., 42, 1101.

6. MELTON, J. T., and LOGUE, B. (1957), Arch. intern. Med. $581-586$.

\section{NOTICE OF SPECIAL INTEREST TO SUBSCRIBERS:}

'WHY NOT HAVE YOUR COPIES OF THIS
JOURNAL BOUND INTO YEARLY VOLUMES?'

You can have your twelve monthly issues fully bound in dark green pin head doth, lettered in gilt on spine with name of Journal, Volume Number and year, complete with index at front, for 22s. 6d. post free. A limited number of out of print journals are available to bind into volumes and make your library complete. Price on application siving details of issues required to complese bsck volumes.
WHY NOT HAVE YOUR JOURNALS BOUND? 